\title{
SIMULATING DARK CURRENT IN NLC STRUCTURES ${ }^{1}$
}

\author{
Cho-Kuen $\mathrm{Ng}^{2}$, Nathan Folwell, Adam Guetz, Valentin Ivanov, Lie-Quan Lee, \\ Zenghai Li, Greg Schussman, and Kwok Ko
}

\author{
Stanford Linear Accelerator Center, 2575 Sand Hill Road, Menlo Park, CA 94025, USA
}

\begin{abstract}
Dark current generation and capture are of great importance in high gradient accelerating structure R\&D especially for the NLC which aims to operate at $65 \mathrm{MV} / \mathrm{m}$ with specific limits on dark current and RF breakdown rates. Although considerable effort has been devoted to building and testing various types of structures to meet these requirements, few theoretical studies have been done to understand these effects in actual structures. This paper focuses on the simulation of dark current in a NLC test structure for which experimental data are available. The parallel time-domain field solver Tau3P and the parallel particle tracking code Track3P are used together to simulate, for the first time, a dark current pulse to compare with the data measured downstream. Results from SLAC X-band 30-cell constant impedance structure for RF drive pulses with different rise times are presented and discussed.
\end{abstract}

PACS: 41.20.Cv; 02.70.Pt; 42.30Va

Keywords: Particle accelerator; Electromagnetism; Dark current

\section{Introduction}

Next generation linear colliders strive to operate at high gradients to achieve greater efficiency. One limiting factor that prevents the accelerator from reaching high gradients is the generation of dark current. Dark current may lead to beam loading of the accelerator structure and, if captured, may also produce undesirable backgrounds downstream in the detector at the interaction point. Therefore, understanding the mechanism of dark current generation and capture is essential to the successful development of high gradient structures for future colliders. Thus far, dark current has been studied mostly through experiments in which measurements were made during high power tests. There is growing interest in understanding the measured data using simulation in order to gain insight into the conditions and structure properties that lead to dark current generation.

Previous efforts in dark current simulation have focused on cylindrically symmetric structures and treated the accelerating fields in steady state. These assumptions ignore the 3D effects of the fundamental power couplers and the transient effects due to the finite pulse length of the accelerating fields. However, they greatly simplify the simulation and many results have been obtained with this approach [1]-[4]. This paper describes the simulation of dark current in a fully 3D model of the accelerating structure and includes the transient response to a realistic drive pulse. Specifically, we chose the SLAC X-band 30-cell constant impedance structure [5] for our end-to-end simulation which has been made possible by new parallel tools from the DOE SciDAC Accelerator Simulation Project.

Under SciDAC, SLAC has developed a new suite of 3D parallel electromagnetic codes based on unstructured grids for modeling large, complex accelerating cavities and RF structures to high accuracy using high performance computers. The codes for use in dark current simulation include the parallel timedomain field solver, Tau3P [6], and the parallel particle tracking module, Track3P [7]. Coupling Tau3P to Track3P provides the transient fields needed for simulating the structure surface response to an RF drive pulse. Applying this simulation model to the 30-cell constant impedance structure allows us to study, for

\footnotetext{
${ }^{1}$ Work supported by Department of Energy contract DE-AC02-76SF00515.

${ }^{2}$ Correspondence author: E-mail address: cho@slac.stanford.edu (Cho-Kuen Ng)
} 
the first time, the dependence of dark current on pulse rise times so that comparison with available data can be made. At the same time, the transient fields from Tau3P are analyzed to understand the increase in peak surface fields due to shorter rise time and the correlation with enhancement in the dark current pulse.

\section{Dark Current Simulation Model}

The simulation of dark current requires three components: a field solver for determining the electromagnetic fields in the accelerator structure, a physics model to govern the particle emission process when the fields interact with the structure surface, and a particle tracker that computes the trajectory of emitted particles under the influence of imposed fields.

\section{(a) Calculation of electromagnetic fields using Tau3P}

Tau3P is a 3D parallel time-domain solver which uses the discrete surface integrals method for unstructured grids to simulate large, complex accelerator structures. The use of unstructured grids allows for conformal meshing of curved surfaces which is particularly important for determining the peak surface fields to a high degree of accuracy. Parallelization provides the ability to handle large problem size and to speed up long simulation. Both capabilities are needed for modeling an entire structure over the duration of a finite RF pulse.

\section{(b) Particle tracking and surface physics in Track3P}

Track3P is a 3D parallel particle tracking code that computes particle motion through the Lorentz force equation using the Boris scheme [8] and with fields input from SLAC's parallel solvers such as Tau3P and Omega3P. On an unstructured grid, the localization of particle position and the interpolation of fields onto it require special treatment for good accuracy and computational efficiency. For example, we have implemented a uniform spatial subdivision scheme to facilitate the particle searching and work is in progress on developing a binned binary space partitioning scheme that can be an order of magnitude faster.

The surface physics model in Track3P follows closely the emission processes described in the paper by Yamaguchi [1]. Field emitted or primary emissions are treated according to the standard FowlerNordheim formula [9] where the emission current is determined by the strength of the surface electric field. Secondary emissions are governed by the Lye and Dekker formula [10] which provides the yield of elastically as well as inelastically scattered electrons, and also true secondaries depending on the energy of the incident electron.

\section{(c) Coupling of Track3P to Tau3P}

In order that Track3P receives the instantaneous fields from Tau3P at each time step, the two codes have to be coupled directly. Although, in principle, one can compute and store the Tau3P fields (over a pulse length) beforehand, the data size would be too large for practical purposes. Our approach is to treat the computation like a PIC method but the fields are computed without charges and currents due to the emitted current, so the scheme is not self-consistent. With all parallel particle codes, the main computational issue is load balancing so that work is evenly divided among the processors at all times. Presently static load balancing is used based on the partitioning of the mesh. As the dark current evolves over time, the workload distribution between particles and fields can vary widely which reduces the parallel efficiency. Methods for improvement using dynamic load balancing schemes are under consideration.

\section{Application to 30-cell Constant Impedance Structure}
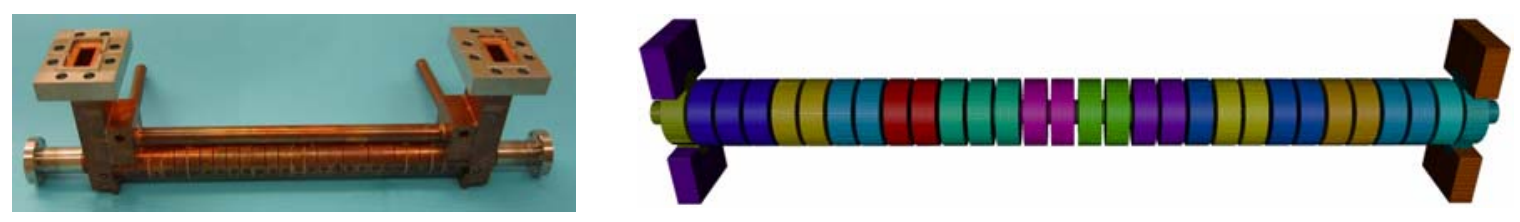

Figure 1: (Left) The X-Band 30-cell constant impedance structure with fundamental couplers, and (right) the corresponding distributed mesh used in Tau3P simulation with different colors representing the portions of mesh being assigned to different processors. 
(a) Peak surface field increase during pulse rise time: The SLAC X-band 30-cell constant impedance structure shown in Figure 1 (iris opening same for all cavities) is a test structure for the NLC and suffered RF damage at the edge of the cell disks during high power tests. It was suspected that RF breakdown was the cause for the damage and is due to extraordinarily high fields sustained at the disk surface. In an effort to understand the origin of these high fields, we drive a pulse with a given rise time and RF power through the structure using Tau3P and monitor the surface electric field around the iris edge of each cell. Figure 2 (left) shows two instances in time of the pulse propagation through the structure. All surface emissions have been turned off. Figure 2 (right) plots the surface electric field at a chosen disk location as a function of time for all 30 cells when excited by a pulse with a 10 ns rise time. It can be seen that, at each cell disk, the field overshoots during the pulse transient before settling down to its expected steady state value. This overshoot is a dispersive effect due to the high frequency content in the rise pulse. When adjusted for the wall loss (which is absent in this simulation) the maximum overshoot is about $17 \%$ over the steady state which is quite a significant increase. Simulation indicates that the increase gets smaller as the rise time gets longer. Since field emission depends on the surface field, this suggests some correlation with experimental data that the peak dark current also decreases with longer rise times.
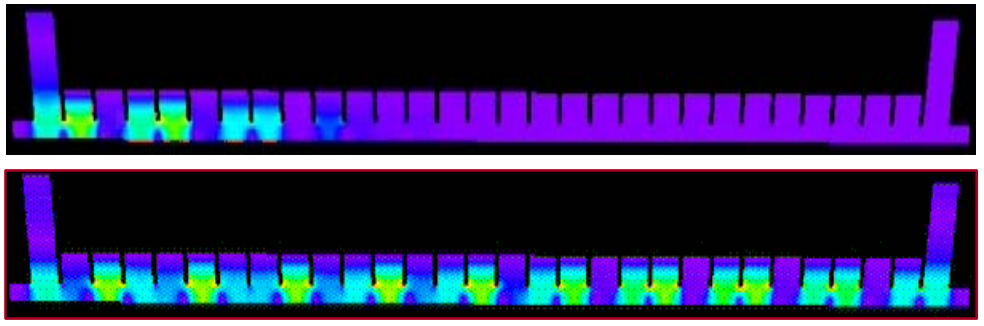

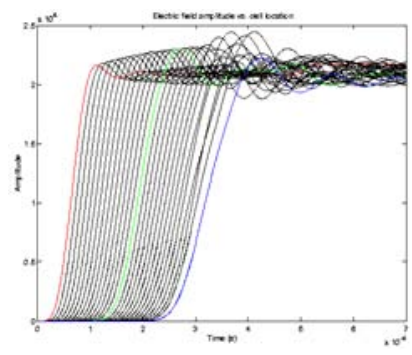

Figure 2: (Left) Two snapshots in time of the pulse propagation in the 30-cell structure; (right) surface field monitored at cell disk in each of the 30 cavities as a function of time, showing overshoot due to the rising pulse (rise time $=10 \mathrm{~ns}$ ).

(b) Generation of dark current pulse: To study dark current, the Tau3P simulation is now coupled to the particle tracking code Track3P which turns on the surface emission processes and tracks the emitted particles (as macro-particles) through the Tau3P fields. These fields are now adjusted by an attenuation factor to account for wall loss and Figure 3 (left) shows the transmitted RF pulse (for three different rise times) monitored at the output waveguide. In Track3P's field emission model, the field enhancement factor $\beta$ is set to 40 . The field emitted (or primary) particles, under the imposed fields, either hit the structure surface generating secondary particles or are captured and accelerated downstream. The simulated dark current is found by collecting all of the particles that exit the downstream beam pipe,. A typical simulation using 30 Pentium-4 processors of a Linux cluster takes 12.6 hours to advance 64,000 time steps for a mesh with about 500,000 hexahedral elements, and the average number of particles at each time step is about 150,000. Meeting the computational requirements and obtaining a fast turnaround time would not have been possible without parallel computing.
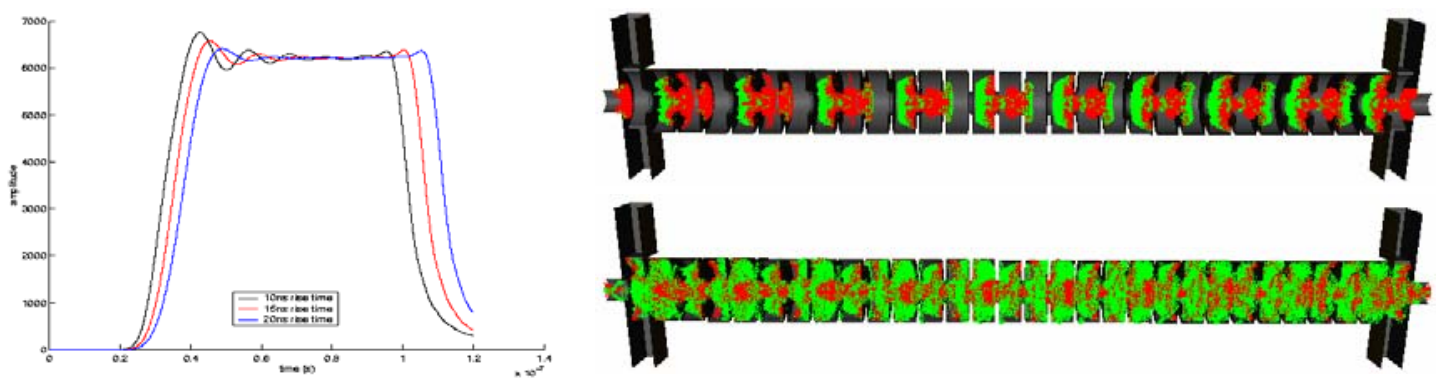

Figure 3: (Left) Transmitted pulse shape monitored at the output waveguide for rise times of 10, 15 and 20 ns respectively; (right) two instances in time of dark current generation with primary particles shown in red and secondaries in green. 


\section{Comparison between Simulations and Experiments}

One of the goals of high power tests is the study of dark current generation because it is recognized that dark current may be the precursor to RF breakdown. In the high power experiment, the dark current pulse is measured downstream of the structure for a range of pulse conditions that include different rise times, various field gradients and pulse lengths [5]. In this work, we focus on a set of data measured in the 30-cell structure that describes the dark current pulse as a function of three pulse rise times of 10, 15 and $20 \mathrm{~ns}$, at a field gradient of $85 \mathrm{MV} / \mathrm{m}$. The data plotted in Figure 4b shows the input, output, and dark current pulses for each case. The same experiment is repeated using Tau3P and Track3P, and the results are summarized in Figure 4a. It shows the computed dark current pulse from the 10 ns case in black, the 15 ns case in red, and the 20 ns case in blue, and that they are in reasonably good agreement with measurement.

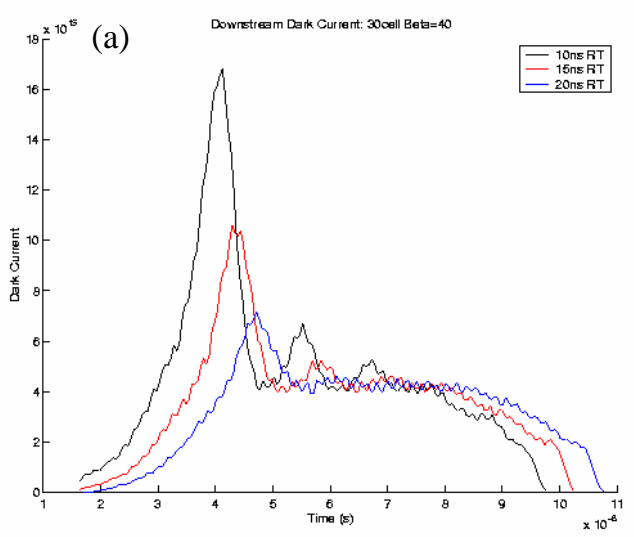

(b)
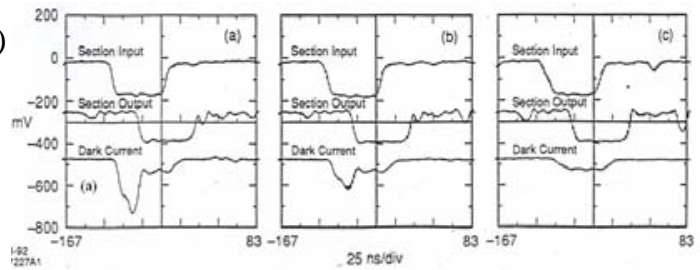

Figure 4: (a) Dark current as a function of time for pulse rise times of 10 (black), 15 (red) and 20 (blue) ns; (b) experimental data for same set of rise times $(10,15$, and $20 \mathrm{~ns}$ from left to right). From top to bottom are the input, output and dark current pulses respectively.

\section{Summary}

This paper reports the first ever dark current simulation of an entire 3D realistic structure, with an actual RF pulse and including secondary emission, using newly developed parallel tools. Good agreement is found between numerical results and measured data on the dark current pulses generated for different rise times in a test structure. The shape of the dark current pulse is corroborated with the RF pulse shape which shows overshoot as a result of dispersive field effects during the rising pulse.

Acknowledgements: We thank Juwen Wang for many helpful discussions.

\section{References}

1. S. Yamaguchi, Simulation Studies of High-gradient Experiments, LAL/RT 92-18, Dec. 1992.

2. P.H. Stolz, M.A. Furman, J.-L Vay, A.W. Nolvik, R.H. Cohen, Phys. Rev., 6(2003) 054701.

3. S. Setzer, W. Ackermann, T. Weiland, M. Krasilnilov, Proc. PAC-2003, P. 3566.

4. K.L. Bane, V.A. Dolgashev, G.V. Stupakov, Simulation of Dark Currents in X-band Accelerator Structures, EPAC’04, Lucerne, Switzerland, July 5-9, 2004.

5. J. W. Wang et al. High Gradient Studies on 11.4 GHz Copper Accelerator Structures, Proc. LINAC’92, p. 716-718, Ottawa, Canada, Aug. 24-28, 1992.

6. M. Wolf, A. Guetz, C.-K. Ng, Modeling Large Accelerator Structures with the Parallel Field Solver Tau3P, Proc. Of $18^{\text {th }}$ Annual Review of Progress in Applied Computational Electromagnetics ACES 2003, Monterey, CA.

7. C. Ng, L. Ge, A. Guetz, V. Ivanov, Z. Li, G. Schussman, Y. Sun, M. Weiner, M. Wolf, Numerical Studies of Field Gradient and Dark Currents in SLAC Structures, ICAP-2002, October 15-18, 2002, East Lansing, Michigan.

8. O. Buneman, Principles and Capabilities of Three-dimensional Plasma Simulation Using Particles, Computational Methods in Applied Science \& Engineering, 5, 529-545 (1982).

9. R.H. Fowler and L. Nordheim, Proc. Roy. Soc. A119, 173 (1928).

10. R.G. Lye and A.J. Dekker, Phys. Rev. 107, 977 (1957). 